\title{
GENERALIZED BICIRCULAR PROJECTIONS VIA RANK PRESERVING MAPS ON THE SPACES OF SYMMETRIC AND ANTISYMMETRIC OPERATORS
}

\author{
AJdA FoŠnER AND DiJANA ILIŠEVIĆ
}

Abstract. We study several related maps from the space of symmetric (respectively, antisymmetric) operators, acting on a complex Hilbert space, to itself: rank preserving linear maps (more precisely, maps preserving rank one operators in the symmetric case, and maps preserving rank two operators in the antisymmetric case), surjective linear isometries and generalized bicircular projections.

Mathematics subject classification (2010): Primary: 47A65, Secondary: 47B49.

Keywords and phrases: Generalized bicircular projections, rank preserving maps, symmetric operators, antisymmetric operators.

\section{REFERENCES}

[1] R. AN, J. Hou, AND L. ZHAO, Adjacency preserving maps on the space of symmetric operators, Linear Algebra Appl., 405 (2005), 311-324.

[2] C. CAO AND X. TANG, Linear maps preserving rank 2 on the space of alternate matrices and their applications, Int. J. Math. Math. Sci., 61-64 (2004), 3409-3417.

[3] T. DANG, Y. Friedman, B. Russo, Affine geometric proofs of the Banach Stone theorems of Kadison and Kaup, Rocky Mountain J. Math., 20 (1990), 409-428.

[4] M. FoŠNER, D. ILIŠEVIĆ, AND C.K. LI, G-invariant norms and bicircular projections, Linear Algebra Appl., 420 (2007), 596-608.

[5] D. ILIŠEviĆ, Generalized bicircular projections on JB*-triples, Linear Algebra Appl., 432 (2010), 1267-1276.

[6] W. KAUP, A Riemann mapping theorem for bounded symmetric domains in complex Banach spaces, Math. Z., 183 (1983), 503-529.

[7] L. Molnár, Selected Preserver Problems on Algebraic Structures of Linear Operators and on Function Spaces, Springer-Verlag, Berlin-Heidelberg, 2007.

[8] J. G. MurPhy, , C*-Algebras and Operator Theory, Academic Press, Boston, 1990.

[9] L. L. STACHÓ AND B. ZALAR, Bicircular projections on some matrix and operator spaces, Linear Algebra Appl., 384 (2004), 9-20. 